\title{
Aspectos ecológicos de flebotomíneos (Diptera: Psychodidae) em área urbana do município de Ponta Porã, Estado de Mato Grosso do Sul
}

\author{
Ecological aspects of Phlebotomines (Diptera: Psychodidae) in the urban area of Ponta Porã \\ municipality, State of Mato Grosso do Sul, Brazil
}

\author{
Paulo Silva de Almeida ${ }^{1}$, Edima Ramos Minzão ${ }^{1}$, Luiz Donizethe Minzão ${ }^{1}$, Silvana Rosa da Silva ${ }^{1}$, \\ Ademar Dimas Ferreira ${ }^{1}$, Odival Faccenda ${ }^{2}$ e José Dilermando Andrade Filho ${ }^{3}$
}

\begin{abstract}
RESUMO
Introdução: Neste trabalho, realizou-se análise faunística de flebotomíneos e levantamento dos índices de infestações (intra e peridomicílio) na área urbana de Ponta Porã/MS, de setembro de 2005 a agosto de 2007. Métodos: As coletas foram realizadas com armadilhas automáticas luminosas do tipo $\mathrm{CDC}$, instaladas mensalmente durante três noites consecutivas, das $18 \mathrm{~h}$ às 6h. Resultados: Foram capturados 3.946 flebotomíneos, pertencentes a oito espécies, com amplo predomínio de Lutzomyia longipalpis, apresentando os maiores índices de frequência, constância, abundância e dominância. Do total capturado, 82,9\% foram de machos e 17,1\% fêmeas. A média mensal de machos capturados $(136,29 \pm 152,01)$ foi significativamente maior que o número médio de fêmeas. Embora não tenham sido constatadas diferenças significativas, verificou-se que a incidência média de flebotomíneos no peridomicílio foi maior do que no intradomicílio. Uma análise de correlação revelou que três variáveis ambientais medidas (temperatura máxima, umidade relativa e precipitação pluviométrica), correlacionaram-se positivamente de forma significativa com a abundância de flebotomíneos. Conclusões: Constitui-se motivo de alerta a predominância de L. longipalpis no município de Ponta Porã, visto que implica na possibilidade de surtos de leishmaniose visceral na área, pois essa espécie é o principal vetor da Leishmania chagasi no estado bem como em outras localidades do Brasil.

Palavras-chaves: Lutzomyia longipalpis. Leishmanioses. Análise faunística. Variáveis ambientais.
\end{abstract}

\begin{abstract}
Introduction: The aim of this study was to carry out an analysis of urban phlebotomine fauna and a survey of infestations (intradomiciliary and peridomiciliary) in Ponta Porã municipality State of Mato Grosso do Sul. Methods: Sand flies were collected with automatic CDC light traps, installed monthly on three consecutive nights, from $18 \mathrm{~h}$ to $6 \mathrm{~h}$, from September 2005 to September 2007. Results: A total of 3,946 phlebotomines, representing eight species, were captured, among which was a large predominance of Lutzomyia longipalpis, presenting high indexes of frequency, constancy, abundance and dominance. Of the total, $82.9 \%$ were males and $17.1 \%$ females. The monthly average number $(136.29 \pm 152.01)$ of males captured being, much larger than that of females. Although the average number of phlebotomines in the intradomicile was much larger than that in the peridomicile, there was no significant statistical difference. A positive correlation was found between the abundance of phlebotomines and the average daily maximum temperature, precipitation and relative atmospheric humidity. Conclusions: The predominance of Lutzomyia longipalpis in Ponta Porã municipality is a reason for concern as regards the possibility of outbreaks of visceral leishmaniasis in the area, because this species is the main Leishmania chagasi vector not only in Mato Grosso do Sul but also nationwide.
\end{abstract}

Key-words: Lutzomyia longipalpis. Leishmaniasis. Faunistic analysis. Environmental variables.

1. Laboratório Regional de Entomologia, Núcleo Regional de Saúde, Secretaria de Estado de Saúde, Dourados, MS. 2. Curso de Ciências Contábeis, Universidade Estadual de Mato Grosso do Sul, Dourados, MS. 3. Coleção de Flebotomíneos, Centro de Referência Nacional e Internacional para Flebotomíneos, Centro de Pesquisas René Rachou, Fundação Oswaldo Cruz, Belo Horizonte, MG.

Endereço para correspondência: Dr. Paulo Silva de Almeida. Lab. Regional de Saúde/NRS/SES. Rua Hilda Bergo Duarte 940, Centro, 79806-020 Dourados, MS.

Tel: 5567 3421-4672; Fax: 5567 3421-4111

e-mail: psilvadealmeida@yahoo.com.br

Recebido para publicação em 10/03/2010

Aceito em 06/10/2010

\section{INTRODUÇÃO}

Os flebotomíneos são insetos noturnos ou crepusculares, tipicamente de matas; porém, devido à ação antrópica, os habitats desses insetos estão sendo modificados, havendo uma redução dos ambientes por eles utilizados. Portanto, as espécies de alguma forma resistem às condições adversas, conseguem explorar novos ambientes, aproximandose cada vez mais dos peridomicílios ${ }^{1-3}$.

O conhecimento local e regional das espécies de flebotomíneos e de seus parâmetros populacionais são importantes, uma vez que esses dípteros são vetores naturais de leishmanioses, doenças consideradas como zoonoses por acometerem primariamente outros animais que não o homem, sendo que este pode estar envolvido de forma secundária ${ }^{4}$.

No Brasil, até o momento, duas espécies estão comprovadamente relacionadas com a transmissão da leishmaniose visceral: Lutzomyia longipalpis (Lutz \& Neiva) e Lutzomyia cruzi (Mangabeira), sendo a primeira considerada como a principal espécie vetora de Leishmania chagasi e a segunda aparecendo apenas em alguns municípios até o momento ${ }^{5}$. Recentemente, L. cruzi foi detectada em 22 municípios do Estado de Mato Grosso ${ }^{6}$. Até o momento, o Estado de Mato Grosso do Sul registrou 39 municípios com a presença de $L$. longipalpis e em cinco municípios, Corumbá, Ladário, Bonito, São Gabriel do Oeste e Camapuã a presença de L. cruzi (PS Almeida: comunicação pessoal, 2009). Além das duas espécies, acima citadas, outras foram recentemente encontradas naturalmente infectadas com Leishmania chagasi ${ }^{7-10}$.

A realização de levantamentos da fauna de flebotomíneoséimportanteparaampliaro conhecimento das áreas de ocorrência desses insetos, subsidiar projetos conservacionistas, visto que a compreensão da dinâmica populacional desse grupo pode se revelar como importante fator para a implantação de políticas de controle epidemiológico ${ }^{11}$. O presente estudo teve como objetivo conhecer a fauna de flebotomíneos em ambiente urbano, verificando sua distribuição sazonal e realizando a análise de parâmetros faunísticos. 


\section{MÉTODOS}

\section{Área de estudo}

O município de Ponta Porã está distante 326km da capital Campo Grande, localizado na região sudoeste do Estado de Mato Gosso do Sul, a $22^{\circ} 32^{\prime} 10^{\prime \prime} \mathrm{LS}$ e $50^{\circ} 43^{\prime} 32^{\prime \prime} \mathrm{LO}$ com uma altitude aproximada de $655 \mathrm{~m}$ acima do nível do mar. O município faz fronteira com o Paraguai e possui uma área estimada em $5.329 \mathrm{~km}^{2}$. Sua população está estimada em 72.207 habitantes $^{12}$. O município de Ponta Porã pertence a bacia hidrográfica do Rio Paraná e possui vegetação característica de floresta estacional semidecidual.

Conforme relatórios obtidos pelo Sistema de Informações de Agravos de Notificação (SINAN), Serviço de Vigilância Epidemiológica da Secretaria Estadual de Saúde de Mato Grosso do Sul, entre 2004 e 2008, o município de Ponta Porã, registrou três casos humanos de leishmaniose visceral (LV) em ambiente urbano e está classificado como área de transmissão esporádica de LV. Com relação à leishmaniose tegumentar, no período de 2007 a 2009, o município de Ponta Porã registrou cinco casos da doença em área rural, sendo três casos no ano de 2008 e dois em 2009.

\section{Coleta dos flebotomíneos}

As coletas foram realizadas com armadilhas automáticas luminosas do tipo CDC, instaladas mensalmente durante três noites consecutivas, das $18 \mathrm{~h}$ às $6 \mathrm{~h}$, conforme preconizado pelo Ministério da Saúde ${ }^{4}$, no período de setembro de 2005 a agosto de 2007. A pesquisa foi realizada em seis pontos fixos (intra e peridomicílio) da área urbana do município de Ponta Porã. O ponto denominado ponto 1 está localizado no bairro Vila Verde, em uma residência próxima a mata ciliar do córrego São João, sendo que a armadilha do peridomicílio foi instalada em um galinheiro distante $50 \mathrm{~m}$ da mata e a segunda armadilha no interior da residência. O ponto 2 localizou-se em uma residência no bairro Vila Dr. Rezende, também próximo a mata e do córrego São João, sendo que a armadilha do peridomicílio foi instalada em um canil, distante $50 \mathrm{~m}$ da mata. $\mathrm{O}$ ponto 3 localizou-se em uma residência no centro da cidade, com a armadilha do peridomicílio também instalada em um canil. O ponto 4 delimitou-se em uma vila militar próximo a uma reserva florestal do exército, com a armadilha do peridomicílio localizada em um galinheiro distante $100 \mathrm{~m}$ da mata. O ponto 5 localizou-se no bairro da Granja, também em uma residência com canil, e o ponto 6 ficou localizado no Jardim Aeroporto, uma residência com galinheiro. Procurou-se instalar as armadilhas do peridomicílio de todos os pontos sempre próximas a um galinheiro ou canil, e a do intradomicílio sempre no interior das residências.

Os insetos foram identificados ${ }^{13}$ e estão depositados na coleção do Laboratório Regional de Entomologia do município de Dourados/MS. A classificação utilizada seguiu a proposta por Galati $^{14}$. Todas as abreviaturas para gênero apresentadas estão conforme Marcondes ${ }^{15}$

\section{Dados meteorológicos}

Os dados climáticos sobre temperatura $\left({ }^{\circ} \mathrm{C}\right)$, umidade relativa do ar (\%) e índice pluviométrico $\left(\mathrm{mm}^{3}\right)$, referentes ao período de estudo, foram obtidos junto ao Instituto Nacional de Meteorologia de Brasília (INMET), Estação Climatológica principal de Ponta Porã/MS.

\section{Análises}

A análise faunística foi baseada nos índices de frequência, constância, abundância e dominância conforme proposto por
Silveira Neto $\operatorname{cols}^{16}$, considerando o número de machos e fêmeas de flebotomíneos capturados nas armadilhas.

A análise de diversidade foi calculada utilizando-se o índice de Shannon-Wiener $(\mathrm{H})$. Para a verificação da riqueza de espécies relacionada ao esforço de coleta, os dados foram interpretados com a curva do coletor através do software Estimates ${ }^{17}$.

Para se verificar a flutuação populacional, foram utilizados os valores médios mensais de temperatura ambiente, de umidade relativa do ar e de precipitação pluviométrica, os quais foram confrontados com dados de abundância mensal dos flebotomíneos. Para se determinar as tendências da relação entre as abundâncias de flebotomíneos e as variáveis ambientais, durante o período de coletas, calculou-se o coeficiente de correlação de Spearman, pois as pressuposições de normalidade das variáveis não foi atendida.

Comparou-se o número médio de indivíduos entre os ambientes, utilizando-se o teste não-paramétrico U de Mann-Whitney, pois as pressuposições exigidas pelo modelo paramétrico não foram atendidas. Todas as análises estatísticas foram realizadas com nível de significância $\alpha \leq 0,05$.

\section{RESULTADOS}

Foram capturados indivíduos representantes de oito espécies, pertencentes a cinco gêneros (Tabela 1). Dos 3.946 indivíduos examinados, $3.271(82,9 \%)$ são machos com uma média mensal de 136,29 (desvio padrão = 152,01) e $675(17,1 \%)$ fêmeas, média mensal de 28,13 (desvio padrão = 31,23), apresentando a razão sexual macho/fêmea de 4,8:1,0. L. longipalpis foi a espécie que apresentou maior variação, entre o número de machos $(83,7 \%)$ e fêmeas (16,3\%) sendo a razão sexual macho/fêmea de 5,1:1,0; seguida por Nyssomyia whitmani (Antunes \& Coutinho) com razão sexual de 2,7:1,0.

Analisando o número total de indivíduos capturados nas armadilhas positivas, a média de machos capturados nas 288 amostras foi de $11,35( \pm 36,89)$ e a média de fêmeas capturadas foi 2,35 $( \pm 7,43)$. Aplicando-se o teste U de Mann-Whitney, verificou-se que houve um número médio de machos significativamente maior $(\mathrm{Z}=-2,66 ; \mathrm{p}=0,008)$, do que o número médio de fêmeas. Diferença observada tanto no intra como no peridomicílio (Tabela 2).

$\mathrm{O}$ índice de diversidade obtido foi $\mathrm{H}^{\prime}=0,175$. Através da análise da curva do coletor (Figura 1), observou-se que os índices (riqueza observada e riqueza esperada) foram sempre próximos ao longo do estudo, indicando que o número de coletas realizadas possibilitou atingir a assíntota, confirmando assim o esforço de coleta realizado.

Análise dos parâmetros faunísticos - em relação a abundância, L. longipalpis apresentou-se como espécie muito abundante, Evandromyia cortelezzii (Brèthes) como espécie rara e as demais espécies foram comuns (Tabela 1). L. longipalpis, Evandromyia sallesi (Galvão \& Coutinho) e N. whitmani apresentaram incidência constante $(w)$, enquanto E. cortelezzii, foi classificada como espécie acessória $(y)$; as demais foram acidentais $(z)$. Lutzomyia longipalpis foi também a única espécie muito frequente $(m f)$ e dominante $(s)$ (Tabela 1).

Verificou-se que a incidência média de flebotomíneos no peridomicílio foi maior do que no intradomicílio; porém, não foram obtidos valores significativos (Tabela 2). 
TABELA 1 - Parâmetros faunísticos dos flebotomíneos nos ambientes intra e peridomicílio encontrados no município de Ponta Porã, Estado de Mato Grosso do Sul, no período de setembro de 2005 a agosto de 2007.

\begin{tabular}{|c|c|c|c|c|c|c|c|c|c|c|c|c|c|c|c|c|c|c|}
\hline \multirow[b]{2}{*}{ Espécie } & \multicolumn{6}{|c|}{ Macho } & \multicolumn{6}{|c|}{ Fêmea } & \multicolumn{6}{|c|}{ Total } \\
\hline & $\mathrm{N}$ & $\mathrm{F} \%$ & F & $\mathrm{C}$ & A & $\mathrm{D}$ & $\mathrm{N}$ & $\mathrm{F} \%$ & $\mathrm{~F}$ & $\mathrm{C}$ & A & $\mathrm{D}$ & $\mathrm{N}$ & $\mathrm{F} \%$ & $\mathrm{~F}$ & $\mathrm{C}$ & A & $\mathrm{D}$ \\
\hline Ev. Lenti & & & & & & & 4 & 0,59 & $\mathrm{pf}$ & $\mathrm{z}$ & c & $\mathrm{n}$ & 4 & 0,10 & $\mathrm{pf}$ & $\mathrm{z}$ & c & $\mathrm{n}$ \\
\hline Ev. sallesi & 2 & 0,06 & $\mathrm{pf}$ & $\mathrm{z}$ & c & $\mathrm{n}$ & 20 & 2,96 & $\mathrm{f}$ & $\mathrm{w}$ & c & $\mathrm{n}$ & 22 & 0,56 & $\mathrm{f}$ & $\mathrm{w}$ & c & $\mathrm{n}$ \\
\hline Ev. cortelezzii & 8 & 0,24 & $\mathrm{pf}$ & $\mathrm{y}$ & $\mathrm{r}$ & $\mathrm{n}$ & & & & & & & 8 & 0,20 & $\mathrm{pf}$ & $\mathrm{y}$ & $\mathrm{r}$ & $\mathrm{n}$ \\
\hline Lu. longipalpis & 3.205 & 97,98 & $\mathrm{mf}$ & $\mathrm{w}$ & $\mathrm{ma}$ & s & 623 & 92,30 & $\mathrm{mf}$ & $\mathrm{w}$ & $\mathrm{ma}$ & $\mathrm{s}$ & 3.828 & 97,01 & $\mathrm{mf}$ & $\mathrm{w}$ & $\mathrm{ma}$ & s \\
\hline Ny. whitmani & 46 & 1,41 & $\mathrm{f}$ & $\mathrm{w}$ & c & $\mathrm{n}$ & 17 & 2,52 & f & y & c & $\mathrm{n}$ & 63 & 1,60 & $\mathrm{f}$ & $\mathrm{w}$ & c & $\mathrm{n}$ \\
\hline Pi. pessoai & 7 & 0,21 & $\mathrm{pf}$ & $\mathrm{z}$ & c & $\mathrm{n}$ & 10 & 1,48 & $\mathrm{pf}$ & $\mathrm{z}$ & c & $\mathrm{n}$ & 17 & 0,43 & pf & $\mathrm{z}$ & c & $\mathrm{n}$ \\
\hline Sc. sordellii & 2 & 0,06 & pf & $\mathrm{z}$ & c & $\mathrm{n}$ & & & & & & & 2 & 0,05 & $\mathrm{pf}$ & $\mathrm{z}$ & c & $\mathrm{n}$ \\
\hline Total & 3.271 & & & & & & 675 & & & & & & 3.946 & & & & & \\
\hline $\mathrm{H}^{\prime}$ & & & & 118 & & & & & & & 0,369 & & & & & & 0,175 & \\
\hline
\end{tabular}

Ev: Evandromyia, Lu: Lutzomyia, Ny: Nyssomyia whitmani, Pi: Pintomyia pessoai, Sc: Sciopemyia sordellii, N: número de flebotomíneos capturadas nas armadilhas, F: frequência, F\%: frequência relativa, C: constância, w: constante, $y$ : acessória, z: acidental, A: abundância, ma: muito abundante, a: abundante, c: comum, d: dispersa, r: rara, D: dominância, s: dominante, n: não dominante, H’: Índice de Shannon-Wiener, pf: pouco frequente, mf: muito frequente, f: frequente.

Analisando-se os dados por pontos, verificaram-se diferenças significativas dos pontos 1 e 2 em comparação com os pontos 3, 4, 5 e 6. Não foram observadas diferenças significativas entre os pontos 1 e 2 e também não se observou diferença significativa entre os pontos 3, 4, 5 e 6 (Tabela 3).

Através do teste de correlação de Spearman, verificou-se que a abundância dos flebotomíneos correlacionou-se positivamente de forma significativa com duas das três variáveis ambientais medidas $\left(\mathrm{r}_{\mathrm{s}}=0,582\right.$; $\mathrm{p}=0,003)$ temperatura máxima $\left(\mathrm{r}_{\mathrm{s}}=0,412 ; \mathrm{p}=0,046\right)$ e umidade relativa.

Quando os fatores físicos analisados foram avaliados por estação, verificou-se que as maiores frequências de flebotomíneos ocorreram no verão-outono de 2006, entre janeiro e março, e início do outono de 2007, no mês de março (Figura 2).
TABELA 2 - Número médio de captura de flebotomíneos (desvio padrão) $\left(\mathrm{n}^{\circ}=\mathbf{2 8 8}\right)$, por gênero e local de captura no município de Ponta Porã/MS, no período de setembro de 2005 a agosto de 2007.

\begin{tabular}{|c|c|c|c|c|c|c|}
\hline \multirow[b]{3}{*}{ Gênero } & \multicolumn{4}{|c|}{ Local de captura } & & \\
\hline & \multicolumn{2}{|c|}{ intradomicílio* } & \multicolumn{2}{|c|}{ peridomicílio* } & \multicolumn{2}{|c|}{ total } \\
\hline & $\mathrm{n}^{\mathrm{o}}$ & $\%$ & $\mathrm{n}^{\mathrm{o}}$ & $\%$ & $\mathrm{n}^{\circ}$ & $\%$ \\
\hline Macho & 9,3 & $25,4 \mathrm{aA}$ & 13,4 & $45,6 \mathrm{aA}$ & 11,4 & $36,9 a$ \\
\hline Fêmea & 2,3 & $6,3 \mathrm{bA}$ & 2,4 & $8,5 \mathrm{bA}$ & 2,4 & $7,4 b$ \\
\hline Total & 5,8 & $18,8 \mathrm{~A}$ & 7,9 & $33,2 a$ & & \\
\hline
\end{tabular}

*letras minúsculas e diferentes nas colunas indicam diferença significativa entre as médias pelo teste de comparação múltipla U de Mann-Whitney, com $\mathrm{p}<0,05$. Letras maiúsculas e iguais nas linhas indicam diferenças não-significativas pelo mesmo teste.

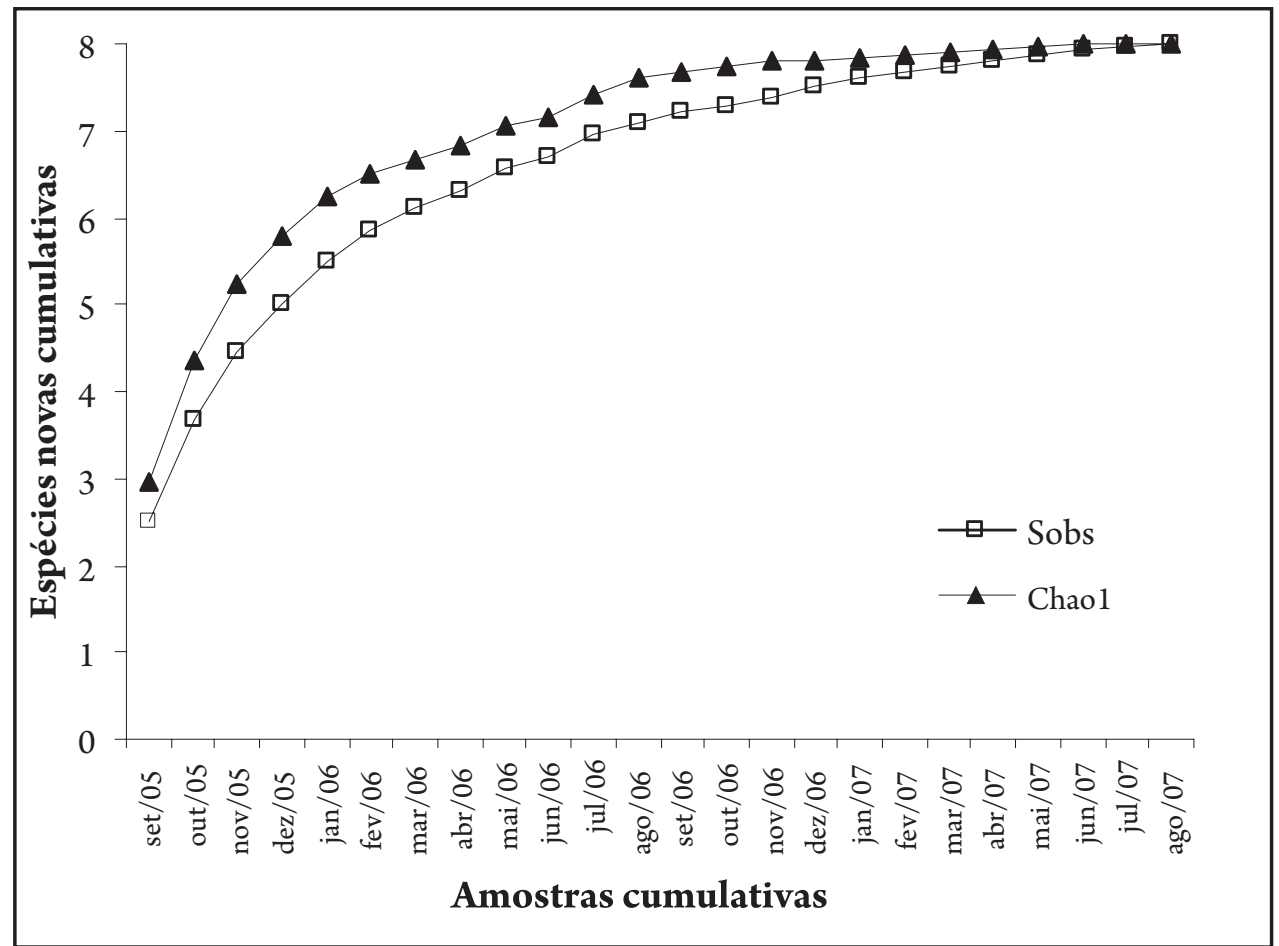

FIGURA 1 - Riqueza observada (Sobs) e esperada (Chao 1) para as espécies de flebotomíneos capturadas com armadilhas luminosas tipo CDC no intra e peridomicílio no município de Ponta Porã-MS (setembro de 2005 a agosto de 2007). 
TABELA 3 - Número, média e desvio padrão de flebotomíneos por ponto de captura no município de Ponta Porã/MS, no período de setembro de 2005 a agosto de 2007.

\begin{tabular}{lcccc}
\hline Pontos & Localidade & Número & Média & Desvio padrão \\
\hline 1 & Bairro Vila Verde & 96 & $16,44 \mathrm{a}$ & 25,308 \\
2 & Vila Dr. Rezende & 96 & $21,79 \mathrm{a}$ & 56,975 \\
3 & Centro da cidade & 96 & $0,85 \mathrm{~b}$ & 2,741 \\
4 & Vila militar & 96 & $1,42 \mathrm{~b}$ & 5,767 \\
5 & Bairro da Granja & 96 & $0,19 \mathrm{~b}$ & 0,621 \\
6 & Jardim Aeroporto & 96 & $0,42 \mathrm{~b}$ & 1,646 \\
\hline Total & & $\mathbf{5 7 6}$ & $\mathbf{6 , 8 5}$ & $\mathbf{2 6 , 9 6 8}$ \\
\hline
\end{tabular}

Letras diferentes nas colunas indicam diferença significativa entre as médias pelo teste de comparação múltipla U de Mann-Whitney, com p $<0,05 / 15$
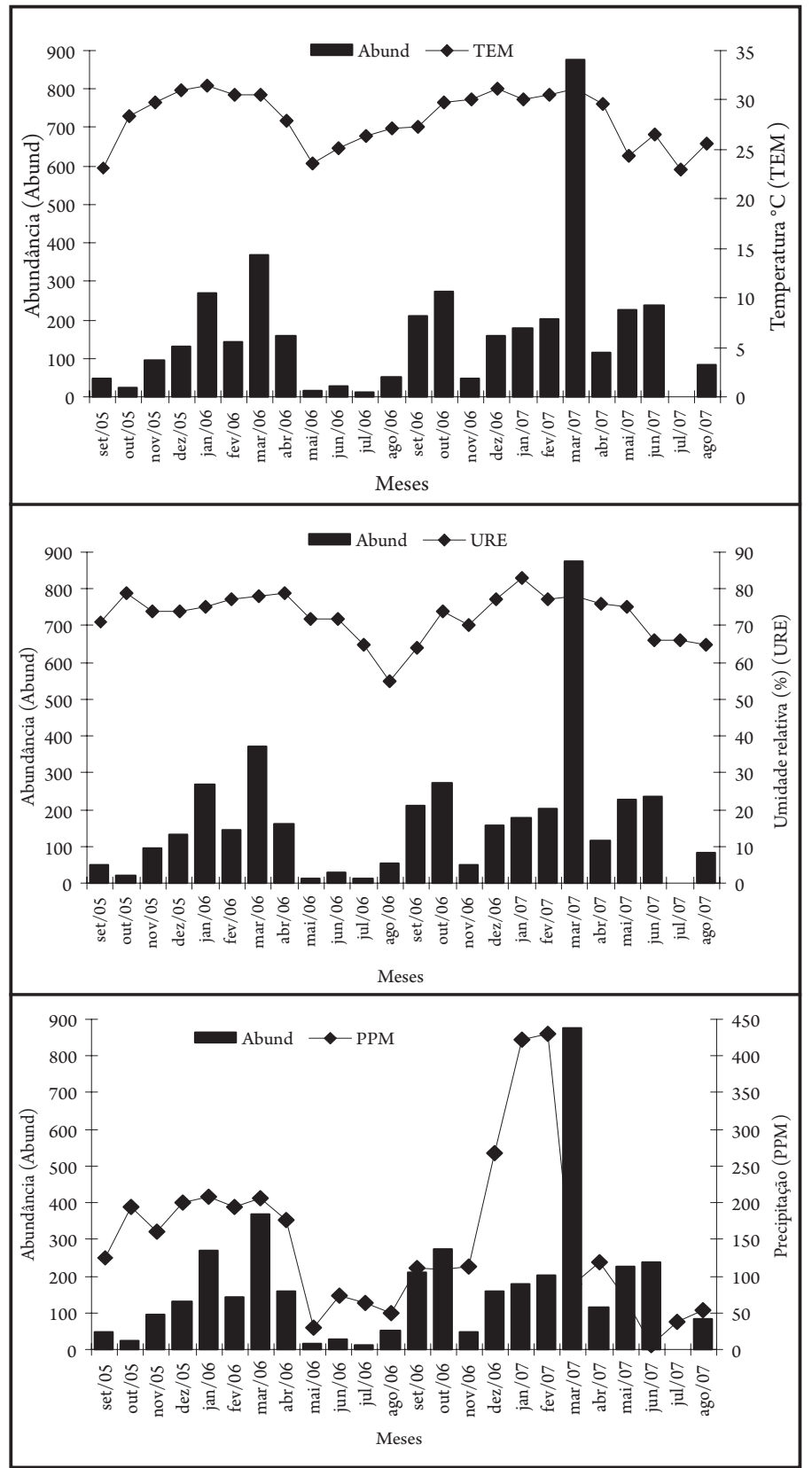

FIGURA 2 - Variação sazonal de flebotomíneos coletados em intra e peridomicílio, na área urbana do município de Ponta Porã, entre setembro de 2005 e agosto de 2007. Os dados de temperatura, umidade e precipitação pluviométrica representam a média mensal.

\section{DIscussão}

Os resultados obtidos demonstraram grande variação entre o números de machos e fêmeas, com destaque para L. longipalpis. $\mathrm{O}$ maior número de indivíduos machos capturados com relação as fêmeas pode ser verificado também em outros estudos ${ }^{18-20}$.

A predominância de L. longipalpis em relação a outras espécies de flebotomíneos também é verificada em outros trabalhos do Estado de Mato Grosso do Sul ${ }^{19,20}$. Estes resultados corroboram os encontrados em Ponta Porã, onde L. longipalpis apresentou ampla predominância, uma vez que esta se destacou com os maiores índices nos parâmetros analisados.

Com relação a incidência de flebotomíneos no intra ou peridomicílio, obteve-se maior número de indivíduos capturados no peridomicílio, todavia essa diferença não foi significativa. Este resultado é corroborado pelos encontrados por Resende cols ${ }^{18}$, que em trabalho realizado em Minas Gerais, observaram não haver diferenças significativas entre intra e peridomicílio.

Embora não tenham sido verificados valores estatísticos significativos, entre a abundância de flebotomíneos e a precipitação pluviométrica, o gráfico de flutuação possibilita a verificação de uma relação desta com o período chuvoso, onde se observam valores crescentes na abundância dos flebotomíneos subsequentes aos picos de chuva. Levando-se em consideração este fato, e que a precipitação pode propiciar uma melhor condição ambiental para larvas e pupas dos flebotomíneos, uma vez que foi trabalhado no estudo com insetos adultos, realizou-se uma análise de correlação com os dados pluviométricos com um mês de defasagem, verificando-se com o mesmo uma correlação positiva e significativa $\left(r_{s}=0,469 ; p=0,024\right)$ entre os dados de abundância e precipitação pluviométrica.

Quanto à sazonalidade, verificou-se maior abundância de L. longipalpis na estação chuvosa. Estes dados também foram observados em várias regiões do Brasil ${ }^{18-22}$.

Analisando-se a curva de sazonalidade de L. longipalpis, obtida neste trabalho, sugere-se para o município de Ponta Porã, a utilização de dois ciclos anuais de aplicação de inseticida com intervalo de três a quatro meses, podendo efetuar o primeiro ciclo no início da estação chuvosa, preferencialmente no mês de setembro e o segundo no mês de janeiro do ano seguinte, conforme recomenda o Manual de Vigilância e Controle da Leishmaniose Visceral ${ }^{4}$.

Os resultados obtidos nos pontos 1 e 2 no município de Ponta Porã estão de acordo com a literatura ${ }^{20}$, no que diz respeito a ecótopos próximos de galinheiros e áreas de matas nativas. A diferença no número de flebotomíneos capturado entre esses pontos e o ponto 4 se deve, provavelmente, à menor distância entre o local de exposição das armadilhas no peridomicílio nos pontos 1 e 2 e a mata quando comparada ao ponto 4.

A ocorrência de algumas espécies de flebotomíneos em áreas urbanas deve-se a capacidade desses insetos de se adaptarem onde ocorreram profundas modificações nos seus habitats naturais provocando a restrição de espaços ecológicos ${ }^{23}$. Como os municípios do Estado de Mato Grosso do Sul, apresentam grandes variações em sua vegetação nativa visto a grande demanda de extensas áreas para a agricultura e a pecuária, e o crescimento urbano em direção às áreas periféricas dos municípios, há um comprometimento das 
áreas naturais antes ocupadas pelos flebotomíneos, os quais têm se adaptado a essas mudanças, e tem sido cada vez mais frequente sua detecção nas áreas periféricas dos centros urbanos.

De acordo com as normas técnicas do Manual de Vigilância e Controle da Leishmaniose Visceral ${ }^{4}$, o município de Ponta Porã é classificado como área de transmissão esporádica de LV, não sendo indicado neste caso, o controle químico do vetor com inseticida residual. Entretanto, é necessário adotar outras medidas preventivas que permitam monitorar o comportamento do vetor e da saúde da população. Missawa e Lima ${ }^{6}$ afirmam que estas medidas preventivas tratam de um conjunto de atividades em saúde pública que engloba as informações necessárias para o conhecimento, sendo possível detectar precocemente as alterações, possibilitando direcionar e orientar a aplicação das ações objetivando atingir o máximo de eficácia.

A presença constante e em abundância elevada de L. longipalpis é motivo de alerta para a possibilidade de surtos de leishmaniose visceral no município de Ponta Porã, visto que essa espécie se apresenta como principal vetor no estado e em outras localidades do Brasil.

\section{AGRADECIMENTOS}

À equipe do Centro de Controle de Zoonoses de Ponta Porã: João Ademar Servin, Marcelino Ferreira da Silva, Raquel Bortolini e Alisson Cordeiro Marques.

\section{CONFLITO DE INTERESSE}

Os autores declaram não haver nenhum tipo de conflito de interesse no desenvolvimento do estudo.

\section{REFERÊNCIAS}

1. Forattini OP, Rabello EX, Galati EAB. Novos encontros de flebotomíneos no Estado de São Paulo, Brasil, com especial referência a Lutzomyia longipalpis. Rev Saude Publica 1976; 10:125-128.

2. Souza GD, Santos E, Andrade Filho JD. The first report of the main vector of visceral leishmaniasis in America, Lutzomyia longipalpis (Lutz \& Neiva) (Diptera: Psychodidae: Phlebotominae), in the State of Rio Grande do Sul, Brazil. Mem Inst Oswaldo Cruz 2009; 104:1181-1182.

3. Santos De Marco T. Determinantes ecológicos da flutuação populacional de flebotomíneos (Diptera: Psychodidae) em área do Parque Nacional do Iguaçu, Estado do Paraná, Brasil. [Dissertação]. [Viçosa]: Universidade Federal de Viçosa; 1997.67p.

4. Ministério da Saúde. Secretaria de Vigilância em Saúde. Departamento de Vigilância Epidemiológica. Manual de vigilância e controle da leishmaniose visceral. Brasília: Ministério da Saúde; 2006.

5. Santos SO, Ribeiro AA, Hoffmann MP, Freitas RU, Malacco MAF. Incrimination of Lutzomyia cruzi as vector of American Visceral Leishmaniasis. Med Vet Entomol 1998; 12:315-317.

6. Missawa NA, Lima GBM. Distribuição de Lutzomyia longipalpis (Lutz \& Neiva, 1912) e Lutzomyia cruzi (Mangabeira, 1938) no Estado de Mato Grosso. Rev Soc Bras Med Trop 2006; 39:337-340.

7. Carvalho GML, Andrade Filho JD, Falcão AL, Lima ACVMR, Gontijo CMF Naturally infected Lutzomyia sandflies in a Leishmania-endemic area of Brazil. Vector-Borne Zoonot Dis 2008; 8:407-414.

8. Pita-Pereira D, Cardoso MAB, Alves CR, Brazil RP, Britto C. Detection of natural infection in Lutzomyia cruzi and Lutzomyia forattinii (Diptera: Psychodidae: Phlebotominae) by Leishmania infantum chagasi in an endemic area of visceral leishmaniasis in Brazil using a PCR multiplex assay. Acta Tropica 2008; 107:66-69.

9. Saraiva L, Carvalho GML, Quaresma PF, Lima ACVMR, Falcão AL, Andrade Filho JD. Natural infection of Nyssomyia neivai (Pinto, 1926) and Evandromyia sallesi (Galvão \& Coutinho, 1939) (Diptera: Psychodidae) by
Leishmania infantum chagasi Cunha and Chagas, 1937 in Minas Gerais, Brazil. J Med Entomol 2009; 49:1159-1163.

10. Savani ESMM, Nunes VLB, Galati EAB, Castilho TM, Zampieri RA, Floeter-Winter LM. The finding of Lutzomyia almerioi and Lutzomyia longipalpis naturally infected by Leishmania spp. in a cutaneous and canine visceral leishmaniases focus in Serra da Bodoquena, Brazil. Vet Parasitol 2009; 160:18-24.

11. Marinho RM, Fonteles RS, Vasconcelos GC, Azevedo PCB, Moraes JLP, Rebelo JMM. Flebotomíneos (Diptera, Psychodidae) em reservas florestais da área metropolitana de São Luís, Maranhão, Brasil. Rev Bras Entomol 2008; 52:112-116.

12. Instituto Brasileiro de Geografia e Estatítica [Internet]. [acessado em nov 2009]. Disponível em http://www.ibge.gov.br/cidadesat/topwindow.htm?1/.

13. Young DG, Duncan MA. Guide to the Identification and Geographic Distribution of Lutzomyia Sand Flies in México, the West Indies, Central and South America (Diptera: Psychodidae). Memoirs of the American Entomological Institute Number 54; 1994.

14. Galati EAB. Classificação de Phlebotominae. In: Rangel EF, Lainson R, editores. Flebotomíneos do Brasil. Rio de Janeiro: FIOCRUZ; 2003. p. 23-51.

15. Marcondes CB. A proposal off generic and subgeneric abbreviations for phlebotomine sandflies (Diptera Psychodidae:Plebotominae) of the world. Entomol News 2007; 118:351-356.

16. Silveira Neto S, Nakano O, Barbin D, Villa Nova NA. Manual de ecologia dos insetos. Piracicaba: Editora Agronômica Ceres; 1976.

17. Colwell RK. Statistical estimation of richness and shared species from samples. Version 6.0b1. User's guide and aplication; 2001. Disponível em http://viceroy. eeb.uconn.edu/estimates/.

18. Resende MC, Camargo MCV, Vieira JRM, Nobi RCA, Porto NMN, Oliveira DL, et al. Seasonal variation of Lutzomyia longipalpis in Belo Horizonte, State of Minas Gerais. Rev Soc Bras Med Trop 2006; 39:51-55.

19. Silva EA, Andreotti R, Honer MR. Comportamento de Lutzomyia longipalpis, vetor principal da leishmaniose visceral americana, em Campo Grande, Estado do Mato Grosso do Sul. Rev Soc Bras Med Trop 2007; 40:420-425.

20. Nunes VLB, Galati EAB, Cardozo C, Rocca MEG, Andrade ARO Santos MFC, et al. Estudo de flebotomíneos (Diptera, Psychodidae) em área urbana do município de Bonito, Mato Grosso do Sul. Rev Bras Entomol 2008; $52: 446-451$

21. Macedo ITF, Bevilaqua CML, Morais NB, Sousa LC, Linhares FE, Amóra SSA, et al. Sazonalidade de flebotomíneos em área endêmica de leishmaniose visceral no município de Sobral, Ceará, Brasil. Ciência Animal 2008; 18:67-74.

22. Rebêlo JMM. Frequência horária e sazonalidade de Lutzomyia longipalpis (Diptera: Psyhodidae: Phlebotominae) na Ilha de São Luís, Maranhão, Brasil Cad Saude Publica 2001; 17:221-227.

23. Ximenes MFFM, Silva VPM, Queiroz PVS, Rego MM, Cortez AM, Batista LMM et al. Flebotomíneos (Diptera: Psychodidae) e Leishmanioses no Rio Grande do Norte, Nordeste do Brasil - Reflexos do Ambiente Antrópico. Neotrop Entomo $2007 ; 36: 128-137$ 